\title{
ACCRETION OF INTERSTELLAR MATTER BY MASSIVE OBJECTS*
}

\author{
E. E. SALPETER $\dagger$
}

Observations of quasi-stellar radio sources have indicated the existence in the Universe of extremely massive objects of relatively small size. The present note discusses the possible further growth in mass of a relatively massive object, by means of accretion of interstellar gas onto it, and the accompanying energy release. Although there is no evidence for (and possibly some evidence against) quasi-stellar radio sources occurring inside ordinary galaxies, for the sake of concreteness we consider the fate of an object of mass $M>10^{6}$ (masses in solar units throughoul, ..1 an ordinary spiral galaxy somewhat like ours.

We first re-examine the hypothetical problem of an object of mass $M$ moving with velocity $U$ (in $\mathrm{km} / \mathrm{sec}$ ) relative to a completely uniform gas medium of density $n$ (expressed as $\mathrm{H}$-atoms per $\mathrm{cm}^{3}$ ) and thermal speed $U_{\mathrm{th}}$. We define (Hoyle and Lyttleton 1939) a characteristic length $s_{0}$ and express the rate of accretion in terms of a dimensionless parameter $\alpha$ to be determined,

$$
\begin{aligned}
s_{0} & =G M / U^{2}=\left(M / U^{2}\right) \times 4.3 \times 10^{-3} \mathrm{pc}, \\
d M / d t & =2 \pi \alpha s_{0}{ }^{2} n U=\alpha M / t_{0}, \\
t_{0} & =\left(U^{3} / M n\right) \times 3.3 \times 10^{11} \text { years. }
\end{aligned}
$$

We assume that the size of the object, as well as the collision mean free path in the gas, is very much smaller than $s_{0}$. For rases with large Mach number, $U \gg U_{\text {th }}$, a stanci , (relative to the object) shock front develr,ps beinind the object, with a large pressure increase across the front. The shape of the shock front is conicai about the accretion axis, as shown schematicaliy in Figure 1,

*Publinhed in The Astrophysical Journal, 140(2): 796-800. This work was oupported in part by contract AFOSR-321-63 with Cornell Univursity.

tGoddard Space Flight Center and Cornell Univeraity, Ithaca, New York.
$N 66 \quad 57950$

but the cone angles depend or the specific heat ratio $\gamma$ of (and on any irreversible energy loss from) the gas. If $\gamma-1 \ll 1$ (almost isothermal compression) then the density increases greatly across the shock front, the cone angles are everywhere small, and pressure gradients parallel to the accretion axis are small compared with gravitational forces. For such cases Bondi and Hoyle (1944) found that $1<\alpha<2$, no matter what the degree of mixing in the shocked region is. For the special case of purely laminar flow, the results are as though molecules moved without collisions except for giving up their transverse momentum when they reach the accretion axis. In this case all the gas with impact parameters less than $\sqrt{ }(2) s_{0}$ is accreted and $\alpha=2$.

If $\gamma-1$ is appreciable, then the cone angles of the shock front are not very small, and the gas molecules lose less of their transverse momentum when crossing the shock front and also regain some longitudinal momentum during the re-expansion in the shocked region (see Ruderman ard Spiegel [1964]). These effects result in $\alpha$ decreasing with

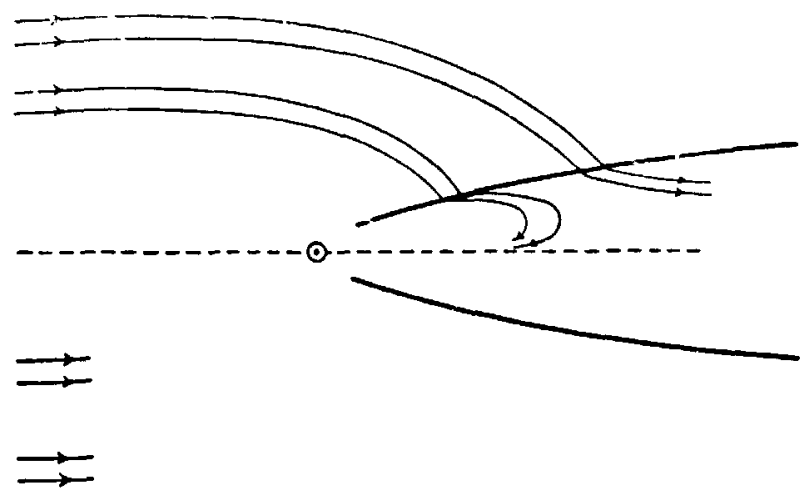

Figure 1.-A schematic view of the flow. 'I he dotted line is the axis of accretion. The heavy line is the standing shock front; the thin lines are (laminar) flow lines. 
increasing $\gamma$. Explicit calculations for $\alpha(\gamma)$ bave not yet been carried out but plausibility arguments lead to the conjecture that $\alpha$ is non-zero for $\gamma<\frac{5}{3}$ and not much less than unity for $\gamma<\frac{4}{3}$. The $\alpha$ is also reduced slightly if $U$ is only slightly larger than $U_{\text {th }}$ (see Bondi [1952] for cases with $\left.U<n U_{\mathrm{th}}\right)$.

McCrea (1953) has discussed the slowing down of an object during its passage through a gas cloud with velocity $U \ll U_{\text {th. }}$. This retardation, analogous to that experienced by a charge moving through a plasma, can be expressed as

$$
d C^{\top} / d t=-\beta U / t_{0}, \quad \beta \approx 1+\ln \left(b_{\max } / s_{0}\right),
$$

where $t_{0}$ and $s_{0}$ are defined in equation (1). For a uniform medium of infinite extent the limiting impact parameter would be $b_{\max } \sim U / \sqrt{ }(4 \pi \rho G)$ as in the plasma problem. In practice the limit in the gravitational problem is the extent of the uniform medium (e.g., thickness of the galactic disk). Consider a massive object in the "halo population" with $U \sim 100$ or 200 (relative to the galactic disk) which spends a fraction of about $(10 / U)$ of its time in the disk. According to equations (1) and (2) the time recuired for slowing down is $(U 10) t_{0} / \phi \beta$ (if ass increase is neglected and $\beta$ assumed consta..c). Unlike accretion of mass, the retardation involves only distant gravitronal encounters and does not require collisions by the "gas molecules," so that the stars in the disk also contribute to the retardation. We assume an average gas density $n \sim 1$ in the disk and $n_{\text {tot }} \sim 10 n$ (including stars) and $\beta \approx 5$. A halo object with initial velocity $U$ (relative to the disk) will slow down in a time less than $10^{10}$ years, roughly the age of our Galaxy, if its mass $M$ cxreeds a critical value of

$$
M_{\mathrm{cr}, \text { al. }} \approx(U / 100)^{4} \times 1.5 \times 10^{6} .
$$

For a highly supersonic object retardation is more rapid than accretion $\left(4 \beta n_{\text {tot }} \gg \alpha n\right)$, an we need to consider accretion only after the object has slowed down to a velocity $U$ not much bigger than $U_{\text {th. }}$. For our large mass $M$ the characteristic impact parameter $s_{0}$ exceeds the "spacing between interstellar gas clouds" (or "turbulence scale length") $d$, and we have to include the random cloud velocities in the thermal velocity. We assume $V_{\text {th }} \sim 10$ or $i \bar{i}, l \sim 25, n \sim 1$. We thus cannot make use of the much lower thermal velocity and higher density available in individual H I region, as atlempted in previous work for smaller masses. On the other hand, the difficulties encountered previously (Schatzman 1955) due to the heating and expansion of $\mathrm{H} I$ regions are absent wher $s_{0}>d$. The energies released by the compression at distances near $s_{0}$ can easily be radiated away at typical $H$ in temperatures, so the compression is almost isothermal ( $\gamma-1$ small). The presence of turbulent magnetic fields in the gas makes the compression more adialatic and raises the effertive $\gamma$ toward $\frac{4}{3}$. The accretion time $\left(t_{0} / \alpha\right)$ is less than $10^{10}$ years if the mass $M$ exceeds

$$
M_{\text {cr, acc. }} \approx(U / 25)^{3}(0.25 / \alpha n) \times 2 \times 10^{6},
$$

and $\alpha$ probably lies between 0.1 and 1 . The characteristic impact parameter is $s=\sqrt{ }(\alpha) s_{0}$ (with $s \approx 7 \mathrm{pc}$ for $M=M_{\text {cr, acc.) and the size of the }}$ object must be less than $s$. The mass of typical halo globular clusters in our own Galaxy is too small for retardation and accretion to become catastrophic by one order of magnitude or so (and their diameter would be sufficiently small).

So far we have only discussed the gravitational capture of gas atoms from the stream at radial distances $r$ from the central object with $r \sim s$, where the energy released per $\mathrm{H}$ atom $(\sim G M H / S$ $\left.\sim \frac{1}{2} H U^{2}\right)$ is only a few eV. If the original gas stream possessed no angular momentum about the accretion axis at all (and $\gamma=\frac{5}{3}$ ) the captured gas would immediately collapse toward the center, somewhat as in star formation but with a continuously increasing mass in the condensation. The evolution of massive stars takes less than $10^{7}$ years and no zero-temperature spherical equilibrium models exist excceding two or three solar masses. After a relativeiy short time, then, most of the accreted matter would have collapsed to the "Schwarzschild singularity" $\left(r=2 \mathrm{GM} c^{2}\right)$. Although the gravitational redshift for radiation from "collapsed matter" is large, note that its "active gravitational" (as well as "passive inertial") m.iss is essentially undiminished. If the original star cluster (or other non-condensed object) exceeded both critical masses in equations (3) and (4), then the accreted collapsed matter eveniually dominates as the cause for further accretion. 
Theories for quasi-stellar (or other condensed) objects which invoke the gravitational collapse of an isolated mass or cluster usually encounter some difficulties in shedding original angular momentum. In our problem the incident "turbulent" gas stream will also carry some small angular momentum at any instant of time, and a captured gas "turbule" will not be able to penetrate to very small radial distances by itself. For our continuous gas stream, however, the angular momentum is only a statistical quantity, provided that the accretion impact pu ameter $s$ is much smaller than characteristic dimensions of the galaxy. The angular momentum then rhanges sign over times of the order of $s / U$ and in about that time a gas "turbule" can shed its angular monentum by collisions with other turbules and spiral in toward the Schwarzschild radius. In this turbulent manner gravitational energy is continually converted into bulk kinetic energy, compressional heating, and compression of turbulent magnetic fields. Densities $n(r)$ at radial distance $r \ll s$ must lie in the range between $(s / r)^{1.5} n$ and $(s / r)^{3} n$ and radiating away energy (bremsstrahlung or synchrotron radliation via energetic electrons) at these high densities presents no great problems.

Fach accreted gas atom eventually approaches the Sichwarzschild radius $r=2 r_{0}$ (with $r_{0}=G M / c^{2}$ $\left.=\left(M / 10^{8}\right) \times 4.710^{-6} \mathrm{pc}\right)$, and we have to estimate the fraction $f_{\text {tot }}$ of its rest-mass energy which is radiated away altogether. As : simplified model for the spiraling in via many collisions, consider a particle in circular orbits with diminishing radii caused by a slow druin of angular momentum and energy. Due to general relativistic effects (see the Appendix) the orbits become unstable to spontaneous spiraling in $a t r=6 r_{0}$. By this time a fraction $f=0.057$ of the rest-mass energy has escaped to infinity. If, at $r=6 r_{0}$, the particle could be brought to rest (by the unlikely collision with a matched purticle of opposite momentumı) an additional fraction $\Delta f=0.126$ could in principle escape. After frec fall to $r=3 r_{0}$ the arditional fraction is 0.22 , and so on. However, the chances of arresting the free fall with appreciable efficiency seem small. In addition, even in the absence of any optical absorption, 50 per cent of all radiation (or highly relativistic particles) emitted isotropically at $r=3 r_{0}$ would be prevented from escaping by general relativistic effects (but only 14.5 per cent at $\left.r=6 r_{0}\right)$. We estimate $j_{\text {tot }}$ (not energy escaping to infinity in all forms) to lie in the range of $0.05-$ 0.20. Note that the gravitational mass of the collapsed source is increased by a fraction 1$f>0.8$ of the accreted mass, and we omit a corresponding correction factor which should be applied to equation (1).

The optical luminosity $L_{\text {opt }}$ can be written in the form

$L_{\mathrm{opt}}=\left(\frac{25}{U}\right)^{3} n \frac{\alpha}{0.25} \frac{f_{\mathrm{opt}}}{0.033}\left(\frac{M}{10^{7}}\right)^{2} \times 2 \times 10^{9} L \odot$

Tf $\alpha$ (and $n$ ) remained constant, then $M$ (and $L$ ) would increase to infinity after a finite time $\left(t_{0}, \alpha\right)$, according to equation (1). However, the effects of radiation pressure decrease the effertive value of the gravitational constant appreciably as $\left(L_{\text {opt }} / M\right)$ approaches a limiting value which depends on the average opacity corfficient. ( $L_{\text {ope }} /$ $M)_{\text {tim }}$ can be as low as 100 (in solar units) in H I-regions in our Galaxy, due to the presence of dust grains (van de Hulst 195i) and as large as $3 \times 10^{4}$ in pure ionizad hydrogen if only Thomson scattering is important. Once the mass $M$ has grown sufficiently for equation (5) to give a value of $\left(L_{\text {opt }} / M\right)$ close to this limit, radiation pressure automatically lowers $\alpha$ in such a way as to keep $L_{\text {opt }} / \boldsymbol{M}$ almost constant. Subsequently, $\boldsymbol{M}$ (and hence $L_{\text {opt }}$ ) increases $w$ ith time only exponentially as $e^{t / \tau}$, where

$\tau=\left(f_{\text {opr }} / 0.03\right)\left(10^{3} M / L_{\text {rpt }}\right) \times 4 \times 10^{8}$ years,

and the characteristic accretion impact parameter $\boldsymbol{s}=\sqrt{ }(\alpha) s_{0}$ increases only as $\sqrt{ } \boldsymbol{M}$. For instance, unler typical conditions for our galartio disk with $\left(L_{\mathrm{spl}} / M\right)_{\mathrm{i} \text { im }} \sim 200$, the exponential growth would start at $M \sim 10^{7}$ and $s$ would reach about $350 \mathrm{pe}$ when $M \sim 10^{9}$ and $I_{\text {opt }} \sim 2 \times 10^{11}$.

To summarize the situation for a highly evolved spiral galaxy like ours (with only a few per cent of the mass remaining in the form of gas): Objects of the order of $10^{n} M_{\odot}$, more thiuis typical globular cluster masses, are require! to initiate a catastrophic aceretion process. Unlike theories involving the gravicutional collapse of solated objects, the time scale of our process aever becumes very short due to the self-limiting effects of radiation pressure. In our Galaxy, in fuct, dust grains would keep the time scale well above $10^{\text {s years, }}$ 
which is much longer than required for guasistellar objects, and the luminosity too low $\left(<10^{12} L_{\odot}\right)$. The situation is likely to be more favorable in systems less evolved than our Galaxy where $(a)$ a larger fraction of the mass is still in the form of gas and $(b)$ the relative abundance of heavier elements, and hence of dust grains, is lower. This leads to a shorter time scale and a larger limiting value for the luminosity-mass ratio for the accreting condensation.

I am indebted to Drs. R. P. Feynman, M. Ruderman. M. Schwarzschild, E. Spiegel, L. Spitzer, and L. Woltjer for helpful criticism and suggestions. In fact, they have contributed most of the positive ideas in this note without being responsible for any of the unwarranted conjectures. I am also grateful to the National Academy of Sciences for a senior postdoctoral fellowship.

\section{APPENDIX}

We use the usual Schwarzschild metric outside of a mass $M$ (and put $c=1$ ),

$$
d s^{2}(1-2 u) d t^{2}-(1-2 u)^{-1} d r^{2}-r^{2} d \theta^{2},
$$

where $u=G M / \mathrm{r}$, and we consider the motion of a single test particle of unit mass in this field. As discussed by Feynman in unpublished lecture notes, the following quartities are constants of the motion

$$
k \equiv(1-2 u) t^{(1)}, \quad l \equiv r^{2} \theta^{(1)},
$$

where a superscript (1) denotes differentiation with respect to proper time. The ierms $l$ and $k$ are generalizations of angular mol zentum and energy, respectively, and are also conserved in any local collision processes (any number of particles at the same $u$ ). The radial distance $r$ satisfies the equation

$$
\left[r^{(1)}\right]^{2}=k^{2}-1+2 u-(1-2 u) l^{2} u^{2} .
$$

The circular orbit with the smallest radius which is stable is then at $u=\frac{1}{8}$ with $l=2 \sqrt{ } 3$ and $k=\sqrt{\frac{8}{9}}$. A photon (or an extremely relativistic particle) can have an unstable circular orbit at $u=\frac{1}{3}$. A particle at rest at $: a$. unce $r(r[1]=l=0$ ) has $k=\sqrt{ }(1-2 u)$. Consider a particle which started at rest far away $(k=1, u=0)$ and ends up at some finite distance from the source with ...ne value of $k$. If the energy reieased luring change all escapes "to infinity" then the escaped energy (after all redshift corrections) is simply $(1-k) c^{2}$.

\section{REFERENCES}

Bondi, H. 1952, M.N., 112, 195.

Bondi, H. and Hoyle, F. 1944, M.N., 104, 273.

Hoyle, F., and Lytmeton, R. A. 1939, Proc. Cambridge Phil. Soc., 35, 405.

Huist, H. C. van de. 1955, Mem. Soc. R. Sci. Liège, 15, 393.

McCr5a, W. H. 1953, M.N., 113, 162.

Ruderman, M., and Spiegel, E. 1964, unpublished.

Schatzman, E. 1055, Gas Dynamics of Casmic Clouds (I.A.U. Symposium No. 2 (Amsterdam: North-Holland Publishing Co.l), p. 193. 